\title{
Uso de internet e mídias sociais por estudantes universitários: um campo de estudo emergencial
}

\section{Use of internet and social media by university students: an emerging study field}

\section{Uso de internet y redes sociales por estudiantes universitarios: un campo de estudio emergente}

\author{
Ilana Luiz Fermann, ORCID 0000-0002-0768-1211 \\ Bianca Ledur, ORCID 0000-0003-4417-0581 \\ Emanueli Ribeiro Beneton, ORCID 0000-0002-6787-8737 \\ Marina Schmitt, ORCID 0000-0002-5840-6228 \\ Juliana Goulart Chaves, ORCID 0000-0003-2754-9979 \\ Ilana Andretta, ORCID 0000-0002-5537-5120
}

\section{Universidade do Vale do Rio dos Sinos (Unisinos), Brasil}

\begin{abstract}
Resumo: Este estudo teve como objetivo mapear o perfil de jovens universitários e a forma como fazem uso de mídias sociais. Trata-se de um estudo descritivo, quantitativo e transversal, realizado em uma universidade privada do Sul do Brasil, com a participação de 143 alunos. Os resultados apontaram que a prática de atividades físicas foi relata por menos da metade dos participantes. Poucos relataram checar as mídias sociais antes de dormir. Quanto as redes e mídias sociais, todos os participantes relataram fazer uso diário do WhatsApp; no que diz respeito a dependência de internet a maioria apresenta dependência leve a moderada. Foi possível concluir que a as mídias sociais embora sejam facilitadoras da comunicação, influenciam diretamente a forma como as pessoas se relacionam presencialmente. Sugere-se que estudos futuros mapeiem a relação e a influência das mídias sociais na vida de universitários, assim como intervenções que auxiliem os mesmos.
\end{abstract}

Palavras-Chave: universitários; internet; mídias sociais

Abstract: This study aimed to map the profile of young university students and the way they use social media. This is a descriptive, quantitative and cross-sectional study carried out at a private university in Southern Brazil, with the participation of 143 students. The results showed that the practice of physical activities was reported by less than half of the participants. Few reported checking social media before going to bed. As for social networks and media, all participants reported making daily use of WhatsApp; with respect to Internet addiction, the majority presents mild to moderate addiction. It was possible to conclude that social media, although they are facilitators of communication, directly influence the way people relate to each other in person. It is suggested that future studies map the relationship and influence of social media in university students' lives, as well as interventions that help them.

Keywords: university students; internet; social media 
Resumen: Este estudio tuvo como objetivo mapear el perfil de los estudiantes universitarios y la forma en que utilizan las redes sociales. Este es un estudio descriptivo, cuantitativo y transversal realizado en una universidad privada en el sur de Brasil, con la participación de 143 estudiantes. Los resultados mostraron que la práctica de actividades físicas fue reportada por menos de la mitad de los participantes. Pocos informaron haber revisado las redes sociales antes de acostarse. En cuando a las redes sociales, todos los participantes informaron que hacen uso diario de WhatsApp. Con respecto a la adicción a internet, la mayoría tiene una adicción leve a moderada. Se concluyó que las redes sociales, aunque facilitan la comunicación, influyen directamente en la forma en que las personas se relacionan en persona. Se sugiere que estudios futuros mapeen la relación y la influencia de las redes sociales en la vida de los estudiantes universitarios, así como las intervenciones que los ayuden.

Palabras clave: universitarios; internet; redes sociales

Recebido: 14/08/2019

Aceito: 10/03/2021

Como citar:

Luiz Fermann, I., Ledur, B., Ribeiro Beneton, E., Schmitt, M., Goulart Chaves, J. \& Andretta, I. (2021). Uso de internet e mídias sociais por estudantes universitários: um campo de estudo emergencial. Ciencias Psicológicas, 15(1), e-2389. doi: https://doi.org/10.22235/cp.v15i1.2389

Correspondência: Ilana Luiz Fermann. Universidade do Vale do Rio dos Sinos (Unisinos), Brasil. Email:ilana.fermann@gmail.com

O acesso a cursos superiores em instituições de ensino, vem crescendo exponencialmente ao longo dos anos (Barros, 2015). A população universitária brasileira se caracteriza atualmente pelo predomínio de estudantes brancos, com idades entre 18 e 24 anos, predominantemente matriculados em instituições privadas (Barros, 2015). Neves e Martins (2016) referem que o crescimento do setor privado de educação se dá em maior proporção quando comparado ao setor público, apontando como uma das principais causas o fato das universidades privadas ofertarem em maior escala cursos no período noturno. A oferta de cursos noturnos possibilita aos estudantes a realização de atividades remuneradas no turno inverso. Vargas, Cantorani, Vargas e Gutierrez (2015) em um estudo com 353 acadêmicos, apontam que 82,6\% $(n=289)$ dos universitários, trabalha durante o dia e estuda durante a noite

A fim de conciliar a rotina de trabalho e compromissos relacionados à universidade, os estudantes despendem pouco tempo para atividades de lazer, o que pode influenciar diretamente em sua saúde física e mental (Vargas et al., 2015). Com a finalidade de diminuir os sintomas de estresse e ansiedade provenientes da rotina e da falta de tempo para lazer, muitos estudantes optam por fazer uso de substâncias como álcool e drogas (Zeferino et al., 2015). No entanto, sabe-se que uma das ferramentas utilizadas para pessoas que possuem altos níveis de estresse e ansiedade é a prática de esportes/atividade física. Essa prática, além de ser benéfica para a saúde e a qualidade de vida dos universitários, podem auxiliar e melhorar o desempenho acadêmico (Silva \& Ehrenberg, 2017).

Outro aspecto relevante a saúde da população universitária, refere-se a qualidade e quantidade de sono. Um estudo realizado com 701 estudantes universitários, em uma universidade Federal, no estado de Fortaleza, apontou que $54 \%$ dos participantes consideram a qualidade de seu sono ruim, $62,7 \%$ dos participantes relatou dormir menos de sete horas por noite. Outro resultado importante desse estudo é a existência de uma diferença significativa nos níveis de sonolência de estudantes universitários que estudam no turno da noite em relação aos estudantes universitários 
que estudam no turno do dia, uma vez que os que estudam à noite apresentaram qualidade de sono ruim e excesso de sono durante o dia (Araújo \& Almondes, 2012). Um estudo realizado com 169 universitários do curso de medicina, apontou que o uso excessivo de mídias e redes sociais, também influencia diretamente na qualidade e tempo de sono (Moromizato et al., 2017). Verificou-se que $42,8 \%$ dos participantes visualizam suas redes sociais sempre que despertam durante a noite, enquanto 53,3 \% relataram que pela manhã ao acordar sua primeira atividade é conferir as redes sociais, com o principal intuito de comunicarem-se com outras pessoas e colegas de curso (Moromizato et al., 2017).

A comunicação é uma necessidade vital que acompanha o ser humano desde o seu nascimento. Ao longo da vida, o ser humano poderá desenvolver estratégias para se comunicar com o mundo, expressando seus sentimentos, desejos e necessidades. No contexto universitário, a internet é utilizada como uma ferramenta de comunicação e aprendizagem, que tende a facilitar auxiliar o estudante, já que o acesso às informações e conteúdos ocorre de maneira rápida (Moromizato et al., 2017). No entanto, a praticidade na obtenção de tais dados, diminui a busca de informações em livros físicos e automaticamente diminui o hábito da leitura, o que influencia diretamente na linguagem verbal e escrita, utilizada pelos universitários (De Oliveira \& Lira, 2015).

Um estudo realizado com 389 estudantes universitários, com o objetivo de identificar quais redes sociais são mais utilizadas e as características destas redes em uma universidade de Minas Gerais, apontou que 94,3\% dos participantes possuíam perfis em redes sociais e destes, 89,4\% acessavam as redes sociais diariamente. Dentre as redes sociais mais utilizadas, o Facebook predominava (94,3 \%), com a finalidade de entretenimento e interação (Dos Santos \& Lopes, 2013).

Outro estudo realizado com 169 estudantes de medicina, de uma universidade do estado de Sergipe, com o objetivo de identificar se há relação entre os sintomas de ansiedade e depressão, uso de internet e redes sociais, apontou que 98,8 \% utilizam internet diariamente, $47,3 \%$ relataram utilizar a internet através de aplicativos de mensagens, 22,5\% relataram utilizar a internet com objetivo de pesquisar, 18,6 \% exclusivamente utilizavam para redes sociais, $8,5 \%$ para entretenimento, 3,1\% utilizam para a acessar notícias. O WhatsApp foi o aplicativo utilizado com maior frequência, apontado por 94,4\% da amostra (Moromizato et al., 2017).

A internet junto a tecnologia permite que dispositivos como computadores, tablets, celulares, entre outros, mantenham-se conectados e com acesso a uma infinidade de conteúdo. Além de permitir o acesso a notícias mundiais, realização de pesquisas científicas, interação profissional, a internet e a tecnologia permitem comunicação em tempo real de um lugar do mundo ao outro. Diante de tantos avanços proporcionados pela tecnologia, a dependência pelo uso de internet é um dos fatores que tem gerado preocupação para pesquisadores e profissionais da área da saúde. Embora as pessoas estejam conectadas entre si, em grande escala, nas redes sociais, Sá (2012) afirma em seu estudo que, o uso excessivo da internet tende a causar prejuízo no funcionamento social do indivíduo. Esta informação vai ao encontro dos achados de Kuss, Griffiths e Binder (2013), que apontam que alunos viciados em internet tendem a apresentar comportamentos desadaptativos e altos índices de neuroticismo. Sentimentos e comportamentos que envolvam isolamento e solidão são exemplos destes prejuízos, principalmente quando o contato deixa de ser social, pessoal e passa a ser apenas virtual (Sá, 2012).

Com o uso da internet as relações face-a-face entre as pessoas tornam-se cada vez mais distantes, e o isolamento pode iniciar de uma maneira sutil, sem que o indivíduo possua essa percepção. O uso excessivo desses meios de relacionamentos, pode resultar em um tipo de dependência (Sá, 2012). Abreu, Karam, Góes e Spritzer (2008) referem que usuários dependentes de internet podem buscar por meio da rede virtual minimizar sentimentos de tristeza, angústia, desânimo. Young e De Abreu (2011) descrevem em seu manual, a importância de atentar para a dependência de internet como um fator de risco para a saúde mental, uma vez que esta dependência pode se tornar um transtorno. 
Diante do exposto, faz-se necessário investigar as relações entre os universitários, a internet e as mídias sociais, buscando medidas preventivas de sintomas de ansiedade e estresse, por exemplo, nessa população. Dessa forma, intervenções poderão ser realizadas com a finalidade de promover mais qualidade de vida, saúde física e mental para os estudantes que estão em formação acadêmica.

Assim, este estudo teve como objetivo descrever o perfil de universitários que utilizam a internet e mídias sociais, bem como o contexto no qual estão inseridos. Além disso, objetiva-se verificar como os universitários estão utilizando esses meios de comunicação e como se comportam em relação às mídias e interações sociais. Para tal, buscou identificar características das mídias sociais mais utilizadas por essa população e os níveis de dependência de internet e mídias sociais que estes apresentam.

\section{Método}

Trata-se de um estudo descritivo, quantitativo e transversal (Sampieri, Collado \& Lucio, 2013). Considerou-se a população de alunos de uma universidade privada do sul do Brasil durante o primeiro semestre de 2018 (20 mil alunos). Optou-se por uma amostra randomizada aleatória estratificada de 382 participantes, considerando o total de alunos da universidade (20.000). A randomização foi realizada por meio de um sorteio que envolvia todas as turmas dos cursos de graduação presencial desta Universidade, selecionando as turmas até completar o número de aluno proposto pela amostra. As turmas foram contatadas pelo professor, que deveria aceitar a participação, caso algum professor rejeite o sorteio iria ocorrer novamente até completar o número de alunos. Como critérios de inclusão considerou-se que os universitários deveriam estar matriculados em algum dos cursos de nível superior e possuir mais de 18 anos.

Os instrumentos utilizados foram (I) Questionário Sociodemográfico e de Uso de Mídias Sociais: desenvolvido pelo grupo responsável pela pesquisa cujo intuito foi identificar as características, como sexo, idade, curso de graduação, mídias sociais que utilizam e hábitos de sono dos participantes. O instrumento Critérios de Classificação Econômica Brasil (Associação Brasileira de Empresas de Pesquisa, ABEP, 2015) foi inserido a este questionário e objetiva identificar os dados socioeconômicos da amostra; (II) Internet Addiction Test (IAT, Young 1998, adaptado por Conti et al., 2012): instrumento cuja proposta é avaliar, através de uma escala Likert de cinco pontos, 20 afirmativas acerca de como a vida do participante pode ser afetada pelo uso excessivo de internet. Este instrumento tem ponto de corte com níveis de dependência de internet como normal (20-30), leve (31-49), moderada (50-79) e grave (80-100) (Conti et al., 2012); (III) Adaptação dos critérios diagnósticos do Transtorno por Uso de Álcool do Manual Diagnóstico e Estatístico de Transtornos Mentais, quinta edição (DSM-5; American Psychological Association, APA, 2014) para compreender dependência de internet. Fora realizada a adaptação através dos 11 itens que compõem os critérios, alterando o uso de álcool por uso de mídias sociais. Por ser uma adaptação não validada cientificamente não possui ponto de corte, ficando apenas como uma descrição para análise de itens separados.

Esta pesquisa teve aprovação do Comitê de Ética da Universidade (CEP 2.289.637) e após o recebimento da carta de anuência da universidade, iniciou-se a coleta de dados, que está ocorrendo por três semestres. Para tal, contou-se com uma equipe de pesquisadoras previamente treinadas que foram nas salas de aula das turmas sorteadas após a autorização dos professores responsáveis. Assim, entregou-se os formulários, instrumentos e duas vias do Termo de Consentimento Livre e Esclarecido (TCLE) aos universitários que concordaram em participar, para que estes fossem entregues preenchidos na semana seguinte, quando as pesquisadoras voltassem à sala para recolhêlos. Ao final do primeiro semestre de coleta, no período de março à julho de 2018, foram contabilizados 143 participantes. Para a análise de dados foi utilizado o programa estatístico 
Statistical Package for the Social Sciences (SPSS), e realizadas análises estatísticas descritivas de frequências e médias.

\section{Resultados}

Obteve-se um total de 143 participantes, sendo a maioria do sexo feminino, com média de idade de 23,7 $(D P=7,9)$ anos e majoritariamente solteira. A maioria dos indivíduos que compõem a amostra, moram com mais alguma pessoa e as pessoas apontadas como referência foram o pai ou a mãe (maiores detalhes estão apresentados na Tabela 1.

Conforme a classificação econômica brasileira, a maioria dos participantes foi considerada como pertencente a classe $\mathrm{B}(\mathrm{ABEP}, 2015)$. No que diz respeito à saúde mental, os participantes afirmaram por meio de auto relato que fazem uso de medicamento psiquiátrico $(12,6 \%, n=18) \mathrm{e}$ $17,5 \%(n=25)$ afirma fazer psicoterapia. Quanto a possíveis avaliações diagnósticas, $14(9,8 \%)$ participantes afirmaram já possuir algum diagnóstico de transtorno de ansiedade e/ou depressão. Demais dados do perfil dos universitários foram descritos na Tabela 1.

Tabela 1.

Perfil sociodemográfico dos universitários

\begin{tabular}{|c|c|c|}
\hline Características dos universitários & $n$ & $\%$ \\
\hline \multicolumn{3}{|l|}{ Sexo } \\
\hline Mulheres & 102 & 71,3 \\
\hline Homens & 41 & 28,7 \\
\hline \multicolumn{3}{|l|}{ Idade } \\
\hline$<25$ & 114 & 80,3 \\
\hline$>25$ & 28 & 19,7 \\
\hline \multicolumn{3}{|l|}{ Estado Civil } \\
\hline Solteiro & 122 & 85,9 \\
\hline Casado & 18 & 12,7 \\
\hline Separado/divorciado & 2 & 1,4 \\
\hline \multicolumn{3}{|l|}{ Mora com quantas pessoas } \\
\hline Sozinho & 5 & 3,6 \\
\hline + uma pessoa & 27 & 19,7 \\
\hline+ duas pessoas & 44 & 32,1 \\
\hline+ três pessoas & 42 & 30,7 \\
\hline + quatro pessoas & 16 & 11,7 \\
\hline \multicolumn{3}{|l|}{ Classificação econômica } \\
\hline $\mathrm{A}$ & 24 & 18,8 \\
\hline $\mathrm{B}$ & 74 & 57,8 \\
\hline $\mathrm{C}$ & 28 & 21,9 \\
\hline $\mathrm{D}$ e $\mathrm{E}$ & 2 & 1,5 \\
\hline \multicolumn{3}{|l|}{ Pessoa de referência } \\
\hline Pai & 31 & 31,7 \\
\hline Mãe & 19 & 30,6 \\
\hline \multicolumn{3}{|l|}{ Trabalha } \\
\hline Sim & 104 & 72,7 \\
\hline Não & 39 & 27,3 \\
\hline \multicolumn{3}{|l|}{ Trabalho remunerado } \\
\hline $\operatorname{Sim}$ & 98 & 68,5 \\
\hline Não & 15 & 10,5 \\
\hline
\end{tabular}


Quanto a prática de esporte, os participantes não apresentaram predominância por uma atividade específica, 47,6 \% $(n=68)$ afirmaram praticar algum esporte, que se derivou entre academia $(n=19,27,9 \%)$, corrida $(n=7,10,3 \%)$ e futebol $(n=4,5,9 \%)$. A frequência com maior predominância foi de até 3 vezes na semana $(n=21,31,3 \%)$. No que diz respeito ao sono dos indivíduos, 32,4\% $(n=46)$ afirmaram dormir de 6 a 7 horas durante a semana e, 43,4 \% $(n=62)$ afirmaram dormir mais de 8 horas nos finais de semana. Sobre a qualidade do sono, $38 \%(n=54)$ afirmaram dormir por tempo suficiente e de forma profunda, e 36,6 \% $(n=52)$ dormem por pouco tempo, porém com um sono profundo. Ainda sobre a os aspectos relacionados ao sono, $39 \%(n=$ 55) dos participantes afirmaram que as utilizam antes de dormir por 30 minutos ou menos e, 51,8 \% $(n=73)$ referiram que utilizam as mídias sociais ao acordar.

Na tabela 2, são apresentadas as informações acerca do âmbito universitário, prezando a não identificação pessoal dos participantes.

Tabela 2.

Características do contexto universitário

\begin{tabular}{lcc}
\hline $\begin{array}{l}\text { Características do contexto } \\
\text { universitários }\end{array}$ & $n$ & $\%$ \\
\hline Localização & & \\
$\quad$ Polo região metropolitana & 112 & 78,3 \\
$\quad$ Polo capital & 31 & 21,7 \\
\hline Turno & 18 & \\
Diurno & 115 & 12,9 \\
Noturno & 6 & 82,7 \\
Diurno e noturno & & 4,4 \\
\hline Escola & 47 & \\
Saúde & 12 & 35,1 \\
Humanidades & 24 & 9,0 \\
Direito & 13 & 17,9 \\
Gestão e negócios & 27 & 9,7 \\
Indústria criativa & 21 & 12,7 \\
Politécnica & & 15,7 \\
\hline Semestre & 81 & 59,1 \\
$1^{\circ}$ ao $4^{\circ}$ & 56 & 40,9 \\
$5^{\circ}$ ao $9^{\circ}$ & & \\
\hline
\end{tabular}

Sobre as mídias sociais, é importante frisar que todos os universitários faziam uso de pelo menos uma mídia social, sendo o WhatsApp acessado por $100 \%(n=143)$ dos participantes, seguida pelo Facebook $(94,4 \%, n=135)$ e YouTube $(93 \%, n=133)$. Quanto à finalidade da utilização de tais mídias sociais, os resultados foram diversos. Porém, cabe ressaltar que para cada mídia social, foi referido um tipo de uso diferente, de acordo com uma escala Likert de 5 pontos, sendo de 0 nunca a 4 sempre. Por exemplo, o Facebook $(M=3,42, D P=1,16)$, WhatsApp $(\mathrm{M}=$ $4,65, D P=0,78)$ e Messenger $(M=2,92, D P=1,34)$ são mais utilizados para "falar ou manter contato com amigos, conhecidos ou colegas de trabalho"; o Pinterest $(M=1,93, D P=1,32)$ e o YouTube $(M=3,40, D P=1,41)$ para "aprender novas coisas"; o Instagram $(\mathrm{M}=3,26, D P=1,50)$ para "acompanhar a vida de pessoas famosas"; Twitter $(\mathrm{M}=2,45, D P=1,50)$, LinkedIn $(M=1,46$, $D P=0,77)$ e Skype $(M=1,38, D P=0,67)$ para "publicar textos e opiniões"; Snapchat $(M=2,13$, $D P=1,35)$ para "publicar fotos suas"; e o Twitch $(M=1,32, D P=0,85)$ para "ver e compartilhar vídeos de outras pessoas". Acerca da privacidade nas mídias sociais, 57,7 \% $(n=82)$ referem que 
tentam manter algum nível de privacidade nos perfis sociais, com 64,5\% $(n=91)$ normalmente aceitando que participem de suas redes virtuais somente pessoas conhecidas.

De acordo com os resultados foi possível constatar que 39,2 \% $(n=56)$ dos participantes checavam as mídias sociais nos intervalos das atividades, 55,9 \% $(n=80)$ dos universitários afirmaram que se sentiam felizes ao acessá-las, 47,9 \% $(n=68)$ declararam fazer de um a três comentários nas redes por dia, e 35,2 \% $(n=50)$ referiram que fazem mais de dez curtidas diárias. Já em caso de publicarem algum comentário que não tenha agradado outra pessoa nos perfis das redes sociais, 48,4\% $(n=15)$ preferia se desculpar pessoalmente, enquanto $23,9 \%(n=34)$ acreditam que seriam capazes de se expressar melhor via redes sociais online se comparado a interação face to face.

Acerca da dependência de internet, os participantes foram avaliados por meio do IAT e de uma adaptação dos critérios do Transtorno de Álcool e outras drogas para dependência de internet do DSM-5 (APA, 2014). É importante destacar que esta adaptação foi realizada pelo grupo de pesquisa, sendo necessário mais estudos para verificar a validade e fidedignidade da adaptação. Os resultados apontam que a maior parte dos alunos apresenta dependência leve a moderada das mídias sociais, conforme apresentado na Tabela 3.

Tabela 3.

Dependência de internet

\begin{tabular}{lcc}
\hline & $n$ & $\%$ \\
\hline DSM 5 & 39 & \\
Nenhuma & 64 & 29,1 \\
Leve & 31 & 47,7 \\
Moderada & 0 & 23,1 \\
Grave & & 0 \\
\hline IAT & 63 & \\
Normal & 51 & 44,1 \\
Leve & 20 & 35,7 \\
Moderada & 2 & 14,0 \\
Grave & & 1,4 \\
\hline
\end{tabular}

\section{Discussão}

Por meio deste estudo, objetivou-se averiguar o perfil sociodemográfico e o uso de mídias sociais de universitários, assim como avaliar as formas de utilização de tais meios de comunicação e a relação entre eles. Os resultados encontrados corroboram com os de outras pesquisas realizadas com a mesma população. Neste estudo, a maior parte dos questionários foi respondida por pessoas do sexo feminino, e isso se confirma na literatura científica internacional (Fernández-Villa et al., 2015; Lima, de Cássia Leiva, \& de Souza Lemes, 2017; Ostovar et al., 2016; Servidio, 2014; Younes et al., 2016). Pode-se considerar este fato como uma limitação ou obstáculo para futuros estudos acerca do uso de internet entre a população universitária, uma vez que o gênero masculino parece ter maior risco de desenvolver adição à internet e sofrer com suas consequências (FernándezVilla et al., 2015; Ostovar et al., 2016; Servidio, 2014).

Ao identificar dados acerca do âmbito acadêmico verifica-se que 82,7 \% $(n=289)$ da amostra estuda a noite, e, que $72,7 \%(n=104)$ possui algum tipo de trabalho. Tais achados corroboram com o estudo de Vargas et al. (2015), o qual identifica em sua amostra que os estudantes frequentam as aulas a noite e trabalham no período diurno. Dos estudantes que afirmaram trabalhar no presente estudo, 68,5\% $(n=98)$ o fazem de modo remunerado. Acredita-se 
que trabalhar durante a graduação esteja associado a situação financeira, visto a necessidade de manter as despesas pessoais e acadêmicas (Neves \& Martins, 2016).

Cabe destacar que no que diz respeito aos dados obtidos sobre hábitos de descanso, evidenciou-se associação entre pior qualidade de sono e uso excessivo de internet (Fernández-Villa et al., 2015; Younes et al., 2016). No presente estudo, averiguou-se que menos da metade da amostra relatou conseguir dormir por 8 horas ou mais diariamente, mesmo nos fins de semana; além disso, pouco mais de $35 \%$ afirmou dormir de forma profunda e por tempo suficiente. A qualidade do sono, interfere em diversos fatores psicossociais, e dormir por períodos curtos tende a aumentar as dificuldades acadêmicas (Andreassen, Torsheim, Brunborg, \& Pallesen, 2012). Além disso, a maioria dos participantes referiu verificar as mídias sociais por pelo menos 30 minutos antes de dormir e ao acordar, o que pode ter influência sob sua qualidade de sono. Dessa forma, sugere-se que estudos futuros investiguem as possíveis associações entre o uso de internet e hábitos de descanso na população universitária.

Nesse estudo quase a metade dos participantes afirmaram praticar ao menos um tipo de esporte ou atividade física, e assim como os hábitos de descanso influenciam na vida dos indivíduos, as atividades físicas também. O estudo de Vargas et al. (2015) verificou que os estudantes despendem pouco tempo para atividades extracurriculares no seu dia-a-dia, o que pode repercutir na saúde mental e física. Silva e Ehrenberg (2017) referem que a atividade física é uma das práticas que pode ser realizada por pessoas com altos níveis de estresse e ansiedade, sendo benéfica para melhorar não só a saúde e qualidade de vida, mas também no desempenho acadêmico.

Acerca das mídias sociais, resultados indicaram que $100 \%$ dos participantes relataram utilizar diariamente o WhatsApp. Este dado corrobora com os resultados encontrados em Sergipe, onde estudantes de medicina relataram, quase com totalidade, fazer uso diário de internet e mídias sociais (Moromizato et al., 2017). Em São Paulo, um estudo com universitários averiguou que mais de $95 \%$ destes utiliza as mídias sociais, e os motivos para tal incluíram diversos benefícios percebidos. Entre eles, a facilidade em obter e trocar informações online e em manter interações e comunicação com pessoas e amigos (Lima, de Cássia Leiva \& de Souza Lemes, 2017). Esse achado vem ao encontro dos dados descritos neste estudo, pois 55,9\% $(n=80)$ dos participantes afirmou sentir algum nível de felicidade ao utilizar a internet.

A partir das análises descritivas realizadas, pôde-se perceber que a porcentagem de universitários que aparentam fazer uso nocivo ou que são considerados dependentes de internet e mídias sociais é pequena, sendo menos de $25 \%$ em relação a adaptação realizada por meio do DSM-5 e menos de $2 \%$ de acordo com o IAT. Esses resultados sugerem que embora haja uma frequente utilização da internet e mídias sociais, os níveis de adição à essas tecnologias ainda não apresentam grande escores. As maiores porcentagens dessa variável foram identificadas como adição leve e normal, respectivamente, DSM-5: 47,8 \%; IAT: 44,3\%, ou seja, a maior parte dos universitários tem nível de dependência de moderado a grave.

Os limites entre o que é real e virtual -offline e online- pode ser considerado na atualidade como pouco rígido. Dessa forma, acontecimentos offline podem repercutir online e vice-versa (Brügger, 2012). Tal perspectiva sugere necessidade de cautela ao tentar compreender o uso de internet e diferenciá-lo da adição, uma vez que, inclusive para universitários brasileiros, a utilização de espaços online como redes sociais faz parte do cotidiano social e acadêmico, podendo substituir livros, livrarias e bibliotecas e facilitando a troca de informações com colegas e professores (Purim \& Tizzot, 2019; Sancovschi \& Kastrup, 2015). Inclusive, no presente estudo, o dado de que todos da amostra fazem uso de alguma mídia social, estabelecido critério de inclusão para participação da pesquisa, corrobora com tais argumentos, indicando que o uso diário e razoavelmente constante de internet pode ser visto como normal e esperado, e não nocivo. Dessa forma, pode-se constatar que, com a disseminação do uso diário da internet e aparelhos smart, é difícil e improvável que as pessoas convivam na sociedade atual sem o contato constante com tais tecnologias (Lima et al., 2017). 
Porém, não é possível afirmar que a utilização cotidiana de espaços online como redes sociais é livre de riscos ou completamente benéfica. Ainda, dentre universitários, o estresse pode ser causa de adição à smartphones (Kuang-Tsan \& Fu-Yuan, 2017), corroborando com a hipótese de que, apesar de cotidiano, o uso de mídias sociais pode escalar para algum prejuízo considerável, como adição às tecnologias da informação. No presente estudo, os dados descritivos, porém, aparentam divergir de tal argumento, uma vez que a porcentagem da amostra que demonstrou adição grave à internet foi minoria. Pesquisas futuras devem atentar, portanto, para tais discrepâncias de hipóteses presentes quanto ao assunto.

\section{Conclusão}

Esse estudo objetivou o mapeamento do perfil de jovens universitário e forma como fazem uso de mídias sociais, a fim de determinar como esses hábitos podem influenciar em suas interações e mecanismos de comunicação sociais. Sabe-se que as mídias sociais fazem parte do cotidiano contemporâneo e que a formas com que as pessoas têm se relacionado sofre influência desses novos modelos relacionais. Dessa forma, o presente estudo identificou o WhatsApp como uma ferramenta unânime com propósito de relacionamento e comunicação, seja com amigos, familiares ou colegas de trabalho. Ou seja, as mídias sociais se apresentam como um facilitador de interações sociais. Tendo em vista que grande parte dos participantes associa sentimentos de felicidade atrelados ao uso das mídias sociais, é possível atentar ao fato de que esse tipo de reforço positivo, junto a sensação de manter contato com um maior número de pessoas, pode incentivar o uso das mídias sociais como um meio de comunicação mais frequente.

Além disso, o estudo identificou que os universitários costumam checar as redes sociais nos intervalos de suas atividades, demonstrando que a busca por esse contato virtual é tão recorrente quanto às interações pessoais face-a-face. Outro aspecto relevante é a relação do uso das mídias sociais junto à aspectos relacionados ao sono, como por exemplo, a necessidade de verificar as mídias sociais ao acordar. Dessa forma, atenta-se ao fato de que as mídias sociais podem adquirir um espaço na vida dos indivíduos que precede as relações de contato físico. Entretanto, cabe atentar para características específicas da população deste estudo, como o fato de a maioria não fazer nenhum tratamento psicológico, o que pode potencializar a evolução de um quadro mais grave de dependência do uso das mídias sociais, uma vez que a consciência sobre a intensidade desses hábitos tem sido pouco exercitada.

Dessa forma, sugere-se que futuros estudos acompanhem o curso da relação dos jovens universitários e as mídias sociais, considerando outras variáveis que possam afetar o exercício social desses indivíduos. Estudos futuros também são indicados para que se tenha maior ciência sobre necessidades de intervenção junto aos universitários e capacitar os profissionais de assistência em relação à possíveis situações de desamparo e manejo das emoções que sofrem interferência desse fenômeno social da contemporaneidade.

\section{Referências}

Abreu, C. N., Karam, R. G., Góes, D. S., \& Spritzer, D. T. (2008). Dependência de Internet e de jogos eletrônicos: uma revisão. Revista Brasileira de Psiquiatria, 30(2), 156-167. doi: $10.1590 /$ S1516-44462008000200014

American Psychological Association (2014). Manual diagnóstico e estatístico de transtornos mentais. Artmed (5a ed.). Porto Alegre: American Psychiatric Association.

Andreassen, C. S., Torsheim, T., Brunborg, G. S., \& Pallesen, S. (2012). Development of a Facebook addiction scale. Psychological reports, 110(2), 501-517. doi:10.2466/02.09.18.PR0.110.2.501-517

Araújo, D., \& de Almondes, K. M. (2012). Qualidade de Sono e sua Relação com o Rendimento 
Acadêmico em Estudantes Universitários de Turnos Distintos. Psico, 43(3), 350-359.

Associação Brasileira de Empresas de Pesquisa (2015). Critério Brasil 2015. Recuperado de: http://www.abep.org/criterio-brasil

Barros, A. S. X. (2015). Expansão da educação superior no brasil: limites e possibilidades. Educação \& Sociedade, 36(131), 361-390. doi: 10.1590/ES0101-7330201596208

Brügger, N. (2012). When the present web is later the past: Web historiography, digital history, and internet studies. Historical Social Research, 102-117. doi: 10.2307/41756477

Conti, M. A., Jardim, A. P., Hearst, N., Cordás, T. A., Tavares, H., \& Abreu, C. N. D. (2012). Evaluation of semantic equivalence and internal consistency of a Portuguese version of the Internet Addiction Test (IAT). Archives of Clinical Psychiatry (São Paulo), 39(3), 106-110.

De Oliveira, G. \& Lira, A. (2015). O uso da internet na aprendizagem de universitários: O que pensam os estudantes de licenciatura? Pensamiento Americano, 8(15),79-98. doi: 10.21803/pensam.v8i15.81

Dos Santos, J. A. \& Lopes, M, D. (2013). A utilização das redes sociais pelos universitários da cidade de Itajubá-MG. Trabalho apresentado no $5^{\circ}$ Encontro Científico Sul Mineiro de Administração, Contabilidade e Economia, Minas Gerais, Brasil, 21-22 Outubro 2013 (pp. 1-10).

Fernández-Villa, T., Ojeda, J. A., Gómez, A. A., Carral, J., Cancela, M., Delgado-Rodríguez, M., \& Moncada, R. O. (2015). Problematic Internet Use in University Students: associated factors and differences of gender. Adicciones, 27(4) 265-275.

Kuang-Tsan, C., \& Fu-Yuan, H. (2017). Study on relationship among university students' life stress, smart mobile phone addiction, and life satisfaction. Journal of Adult Development, 24(2), 109-118. doi: 10.1007/s10804-016-9250-9

Kuss, D., Griffiths, M., \& Binder, J. (2013). Dependência de Internet em estudantes: Prevalência e fatores de risco. Computadores em Comportamento Humano, 29(3), 959-966.

Lima, A. C. A., de Cássia Leiva, J., \& de Souza Lemes, S. (2017). Viver em rede: uma análise sobre as implicações do uso das mídias sociais por estudantes universitários. Revista on line de Política e Gestão Educacional, 896-912. doi: 10.22633/rpge.v21.n.esp1.out.2017.10459

Moromizato, M. S., Ferreira, D. B. B., Souza, L. S. M. D., Leite, R. F., Macedo, F. N., \& Pimentel, D. (2017). O Uso de Internet e Redes Sociais e a Relação com Indícios de Ansiedade e Depressão em Estudantes de Medicina. Revista Brasileira de Educação Médica, 41(4), $497-$ 504. doi: 10.1590/1981-52712015v41n4rb20160118

Neves, C.E.B. \& Martins, C.B. (2016). Ensino Superior no Brasil: uma visão abrangente. Em: T. Dwyer, E. L. Zen, W. Weller, J. Shugang \& G. Kaiyuan (Eds.), Jovens universitários em um mundo em transformação uma pesquisa sino-brasileira (pp. 95-120). Brasila: Ipea.

Ostovar, S., Allahyar, N., Aminpoor, H., Moafian, F., Nor, M. B. M., \& Griffiths, M. D. (2016). Internet addiction and its psychosocial risks (depression, anxiety, stress and loneliness) among Iranian adolescents and young adults: A structural equation model in a crosssectional study. International Journal of Mental Health and Addiction, 14(3), 257-267. doi: 10.1007/s11469-015-9628-0

Purim, K. S. M., \& Tizzot, E. L. A. (2019). Analisys of Undergraduate Medical Students' Use of Facebook. Revista Brasileira de Educação Médica, 43(1), 187-196. doi:10.1590/198152712015v43n1rb20180139

Sá, G. M. (2012). À frente do computador: a Internet enquanto produtora de dependência e isolamento. Sociologia, 24, 133-147.

Sampieri, R., Collado, C. and Lucio, M. (2013). Definição do alcance da pesquisa a ser realizada: exploratória, descritiva, correlacional ou explicativa. (5a ed.). Em: R. Sampieri, C. Collado and M. Lucio (Eds.), Metodologia de pesquisa (pp. 99-110). Porto Alegre: Penso. 
Sancovschi, B., \& Kastrup, V. (2015). Computador-internet nas práticas de estudo contemporâneas: uma pesquisa com estudantes de psicologia. Psicologia: ciência e profissão, 35(1), 83-95. doi: 10.1590/1982-3703001252012

Servidio, R. (2014). Exploring the effects of demographic factors, Internet usage and personality traits on Internet addiction in a sample of Italian university students. Computers in Human Behavior, 35, 85-92. doi: 10.1016/j.chb.2014.02.024

Silva, M. G. Q., \& Ehrenberg, M. C. (2017). Atividades culturais e esportivas extracurriculares: influência sobre a vida escolar do discente. Pro-posições, 28(1), 15-32. doi: 10.1590/19806248-2015-0055

Vargas, L.M., Cantorani, J.R.H., Vargas, T.M., \& Gutierrez, G.L. (2015). Fatores associados ao nível de atividade física entre estudantes universitários. Revista Brasileira de Qualidade de Vida, Ponta Grossa, 7(3), 158-168. doi: 10.3895/rbqv.v7n3.3217

Younes, F., Halawi, G., Jabbour, H., El Osta, N., Karam, L., Hajj, A., \& Khabbaz, L. R. (2016). Internet addiction and relationships with insomnia, anxiety, depression, stress and selfesteem in university students: a cross-sectional designed study. Plos one, 11(9), 1-13. doi: 10.1371 / journal.pone. 0161126

Young, K. S. \& De Abreu, C. N. (2011). Dependência de internet: manual e guia de avaliação e tratamento. Artmed Editora.

Young, K. S. (1998). Internet addiction: The emergence of a new clinical disorder. Cyberpsychology \& behavior, 1(3), 237-244. doi: 10.1089/cpb.1998.1.237

Zeferino, M. T., Hamilton, H., Brands, B., Wright, M. D. G. M., Cumsille, F., \& Khenti, A. (2015). Consumo de drogas entre estudantes universitários: família, espiritualidade e entretenimento moderando a influência dos pares. Texto \& Contexto Enfermagem, 24, 125-135. doi: 10.1590/0104-07072015001150014

Participação dos autores: Participação dos autores: a) Planejamento e concepção do trabalho; b) Coleta de dados; c) Análise e interpretação de dados; d) Redação do manuscrito; e) Revisão crítica do manuscrito.

I. L. F. contribuiu em a, b, c, d, e; B. L. contribuiu em a, b, c, d; E. R. B. contribuiu em a, b, c, d; M. S. contribuiu em a, b, c, d; J. G. C. contribuiu em a, b, c, d; \& I. A. contribuiu em a, e.

Editora científica responsável: Dra. Cecilia Cracco. 